\title{
Experimental study on the particle removal efficiency of pellet flue gas in wire-tube electrostatic precipitators
}

\author{
Cui Yongzhang ${ }^{1,2, a}$, Zhang Linhua ${ }^{1,2, b}$,Sun $\mathrm{Jie}^{1, \mathrm{c}}$, Zhang Guokai ${ }^{1, \mathrm{~d}}$, \\ 1. School of thermal energy engineering, Shandong Jianzhu University, Jinan 250101, China; \\ 2. Key Laboratory of Building Renewable Energy Utilization Technologies, Shandong Jianzhu \\ University, Ministry of Education, Jinan 250101, China \\ aemail:cyz@sdjzu.edu.cn, bemail:zhth0015@sdjzu.edu.cn, cemail:tangmt91@163.com, ${ }^{\text {d }}$ \\ email:13964060604@163.com
}

Keywords: pellet, Particle removal efficiency, electrostatic precipitators, wire-tube

\begin{abstract}
This paper evaluates experimental the influence operating parameters of wire-tube electrostatic precipitators on Particle collection efficiency of pellet furnace flue gas, such as particle diameter, electric field voltage, Particle concentration, and flue gas velocity. Particle removal efficiency increases firstly and then tends to be constant with voltage increasing, and firstly increases and then decreases with Particle concentration, decreases with flue gas velocity.
\end{abstract}

\section{Introduction}

The combustion of biomass is an established technology for the utilization of Bioenergy, Generation of heat by biomass combustion takes advantage of a renewable source of energy and can be help to reduce the overall emissions of $\mathrm{CO}_{2}$. However, biomass combustion can release fine particles from 50 to $500 \mathrm{mg} / \mathrm{m}^{3}$, fine particle diameter are from 1 to $5 \mu \mathrm{m}[1-3]$. It is urgently required to decrease particle emissions.

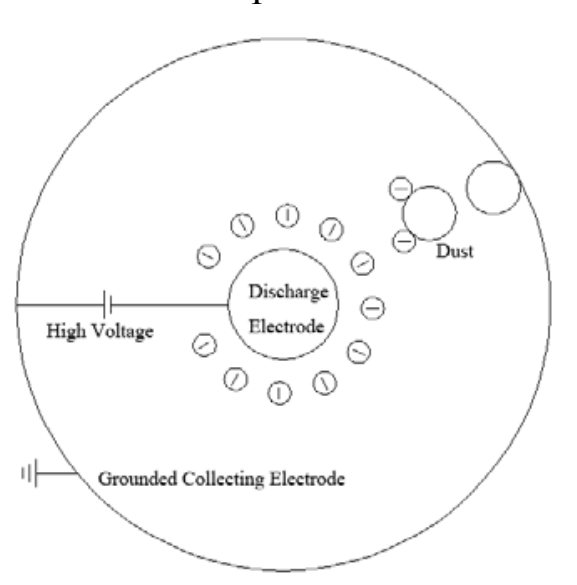

Fig1. Schematic diagram of a wire-tube Electrostatic precipitator

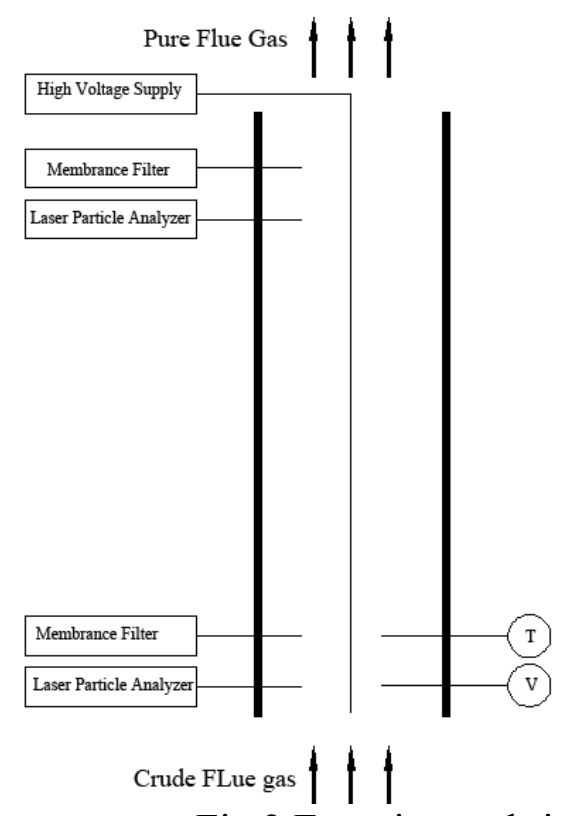

Fig 2 Experimental rig

Particle removal techniques include mechanical collector (cyclone), wet scrubber, fabric filter or baghouse and electrostatic precipitator (ESP) [4-8].For ESP main merits are low pressure drop, suitable for sub-micron particles and suitable for all types of pellet combustors. Particle of biomass pellet is light and low resistivity, so performance can be changed. Experimental test were executed on power polar, power voltage, particle diameter, Particle concentration, and tube length with a pellet boiler prototype. 


\section{Theoretical Model}

The schematic diagram of a wire-tube ESP was illustrated in Fig1. Supplying high voltage between the collecting electrode and discharge electrode generates a corona discharge that produces minus ions. The electrically charged Particles are attracted towards the collecting electrode by an electrostatic force.

The electric field at the discharging wires is E which is given by Peek formula [7]

$$
\begin{aligned}
& E_{0}=f\left(31.02 \delta+0.954 \sqrt{\frac{\delta}{r}}\right) \times 10^{5} \\
& \delta=\frac{\mathrm{M}_{\text {flue }}}{\mathrm{M}_{\text {air }}} \frac{\mathrm{PT}_{0}}{\mathrm{P}_{0} \mathrm{~T}}
\end{aligned}
$$

Where $\mathrm{E}$ is the electric field intensity, $\mathrm{r}$ is wire radius, $\delta$ is gas relativity density, $\mathrm{f}$ is the roughness factor, $\mathrm{M}$ is gas molecule weight.

The migration velocity $\mathrm{v}$ of a particle with a diameter $\mathrm{d}$ is [7]

$$
\begin{aligned}
& \mathrm{v}=\frac{q E C}{3 \pi \mu d} \\
& \mathrm{c}=1+\frac{\lambda}{d}\left[2.514+0.8 \exp \left(-0.55 \frac{d}{\lambda}\right)\right]
\end{aligned}
$$

Where $\mathrm{q}$ is the charge a particle has acquired, is viscosity of flue gas, $\mathrm{c}$ is slip factor, is the mean free path for flue gas, $d$ is particle diameter

The particle concentration inlet and out of ESP was monitored simultaneously with two membrane filter, the Particle removal efficiency $\eta$ was defined as

$$
\eta=\left(1-\frac{C_{\text {in }}}{C_{\text {out }}}\right) \times 100
$$

Where $C_{\text {in }}$ and $C_{\text {out }}$ are inlet and outlet particle concentration.

\section{Experiment set-up}

Experimental rig was shown in Fig 2. Collection tube diameter is $157 \mathrm{~mm}$, and length is $0.8,1.2$ and $1.6 \mathrm{~m}$. Flue gas of fired with cotton pellet was used as a Particle source, particles diameter below $5 \mu \mathrm{m}$. Particle density was measured with membrane filter under certain flow rate at inlet and outlet of wire-tube electrostatic precipitator. Particle diameter was measure with laser particle analyzer. Flue gas temperature and velocity were measure with Pt100 and pitotmeter. High-voltage DC power supply can provide positive and negative voltage of $0-30 \mathrm{kv}$. Flue gas flow rate was from 10 to $50 \mathrm{~m}^{3} / \mathrm{h}$, Particle density was from 50 to $400 \mathrm{mg} / \mathrm{m}^{3}$. Corona electrode is $0.5 \mathrm{~mm}$. 


\section{Result and discussion}

\section{Effect of power polar}

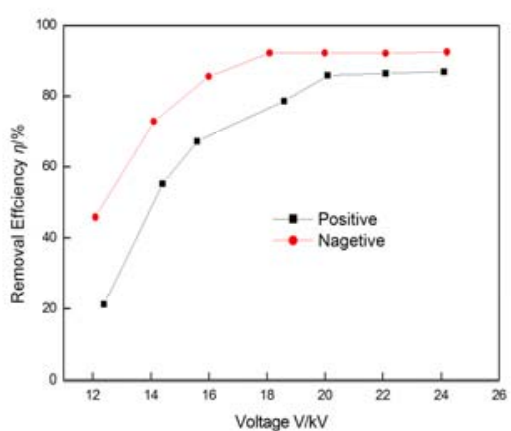

Fig3 Effect of power polar

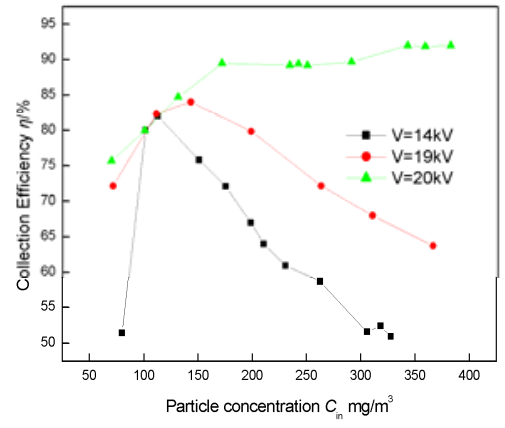

Fig 4 Effect on particle concentration

Under the same experimental conditions, the Particle removal efficiency of negative voltage is always higher $10 \%$ than positive voltage in Fig3. When the discharge electrode is negative, they weak the electric field intensity of collecting electrode side and strengthen intensity toward the tip, so that the entire field evenly distributed. And migrate velocity of free electrons to the collecting plate is much bigger than the positive ions, so at the same voltage, corona electric current of negative corona is much bigger than positive.

\section{Particle concentration}

Test conditions: $\mathrm{v}=0.3 \mathrm{~m} / \mathrm{s}$, Cin $=70-360 \mathrm{mg} / \mathrm{m}^{3}, \mathrm{~V}=14-20 \mathrm{kV}$. As shown in Fig4, particle removal efficiency increases firstly and then decreases under lower power voltage, but particle removal efficiency all increases under higher power voltage. Higher power voltage, higher electric field intensity, higher charged concentration, higher removal efficiency. With the increasing of dust concentration in flue gas, the number of charged dust particles also increased, so that the corona current formed by charged dust particles is not big, but space charge is great, seriously inhibit the generation of corona current, so that dust particles can not get enough charge, resulting in dust removal efficiency decreasing. So higher particle concentration, should higher power voltage and particle should be charged enough.

\section{Power voltage}

Test conditions: $\mathrm{v}=0.35 \mathrm{~m} / \mathrm{s}, \mathrm{Cin}=130-290 \mathrm{mg} / \mathrm{m}^{3}, \mathrm{~V}=12-28 \mathrm{kV}$. As shown in Fig 5 , the particle removal efficiency increases firstly as the voltage increases. For higher voltage, higher particle migrating velocity, higher electric field intensity. However, when the voltage reaches a certain value, $20 \mathrm{kV}$, the efficiency basically remained constantly.

\section{Flue gas velocity}

Test condition: $V=20 \mathrm{kV}, C_{\text {in }}=190 \mathrm{mg} / \mathrm{m}^{3}$. In Fig 6 , flue gas velocity $v$ increases from $0.24 \mathrm{~m} / \mathrm{s}$ to $0.48 \mathrm{~m} / \mathrm{s}$, the particle removal efficiency $\eta$ decrease from $91 \%$ to $76 \%$. As flue gas velocity increases, Flow distribution in tube is more uneven, time of migrating to wall of charged particle is shorten, charge density of particle decrease, migrating velocity is relatively lower.

\section{Conclusions}

From the experimental particle collection efficiency of particle diameter and concentration, voltage and gas velocity, the following conclusions can be reached: (1) The larger the particle diameter, the higher the grade dust removal efficiency. When the particle diameter is larger than $3.1 \mu \mathrm{m}$, the grade dust removal efficiency can reach $100 \%$. (2) Particle removal efficiency of negative voltage is higher $10 \%$ than that of positive voltage. (3) Particle removal efficiency firstly increases with the voltage increasing, and then constantly. Voltage should be higher than 20kV.(4) 
Particle removal efficiency firstly increases with dust concentration and then decreases, decreases with the flue gas velocity increasing.

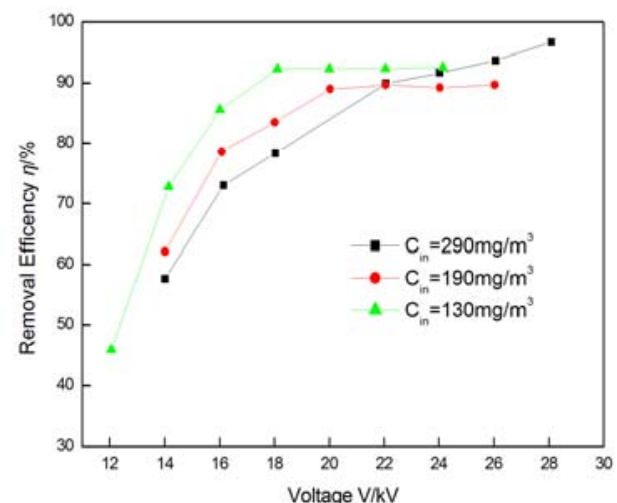

Fig 5 Effect on power voltage

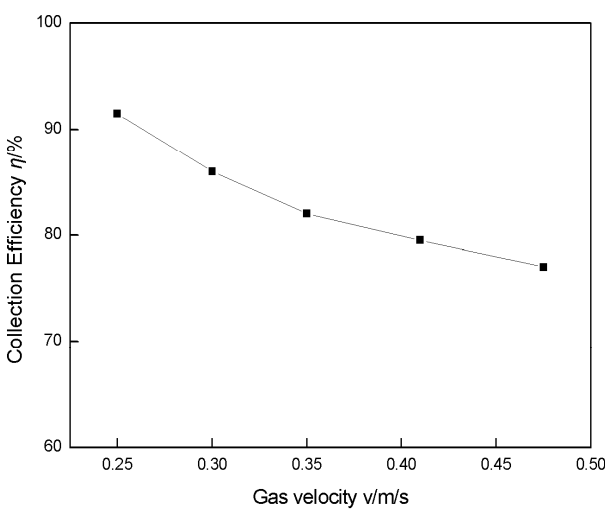

Fig6 Effect on flue gas velocity

\section{Acknowledgement}

This study was funded by the Doctoral Fund Project of Shandong Jianzhu University (XNBS1217) and Scientific and Technological Research Projects in Jinan and Shandong Province (201202087, 2011GNC11401).

\section{References}

[1] U.Fernades, M.Costa, Particle emission form a domestic pellets-fired boiler, Fuel processing technology, 2012, 103:51-56

[2] Kaung M.W Particle and gaseous emissions form realistic operation of residential wood pellet heating system, atmospheric Environment, 2012,59:320-327

[3] Lei Hong, He Ji Cheng, Three-dimensional numerical method for calculating electrical field in the wire-plate electrostatic precipitator, Journal of Industrial heating, 2007,36(6):15-17

[4] Yao Jianjun, Analysis and application of volt-ampere characteristic curve of electric precipitator, Metallurgical power, 2011,143(1):5-7

[5] Zhao Yi, Modelling study on particle precipitator characteristics of ESPs, Environmental science \& technology, 2010, 33(2):68-71.

[6] Jian Bo Zhu. Environmental protection equipment Technology Series, Electrostatic precipitators [M], China electric power press, 2010.

[7] Y. Zhuang, Y. J. Kim. Experimental and theoretical studies of ultra-fine particle behavior in electrostatic precipitators. Journal of Electrostatics, 2000 (48)245-260.

[8] Chen Peng, Study on the influence factor of collection efficiency of the electrostatic precipitator[M], Northeastern University, 2009 